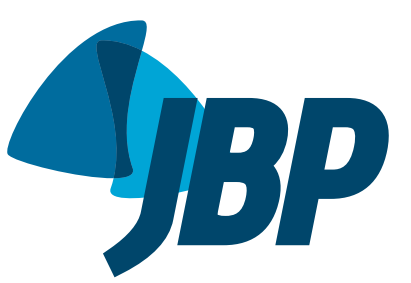

\section{Right lung exclusion in massive pulmonary thromboembolism}

\author{
Rodrigo Abensur Athanazio'1, Samia Zahi Rached ${ }^{1}$
}

A 37-year-old female patient presented to the emergency department with a 3-week history of dyspnea, hypoxemia, pleuritic chest pain, and lower limb edema. She had no history of comorbidities and had had two normal pregnancies. There was no family history of thrombosis. An electrocardiogram showed right axis deviation, and blood tests revealed elevated D-dimer levels. A routine chest X-ray showed oligemia in the right hemithorax and engorgement of the left pulmonary artery (Figure 1). Chest CT angiography confirmed the presence of a thrombus in the pulmonary artery trunk and full occlusion of the right segment (Figure 2). The coronal reconstruction shown in Figure 3 elegantly demonstrates the complete lack of pulmonary perfusion in the right lung, together with ipsilateral oligemia. Echocardiography confirmed pulmonary hypertension (systolic pulmonary artery pressure, $80 \mathrm{mmHg}$ ) and right ventricular dysfunction. Because of hemodynamic instability, the patient was submitted to thrombolysis with alteplase and started on anticoagulation therapy. Her dyspnea persisted, and she was categorized as New York Heart Association functional class III. After 6 months, she evolved to chronic pulmonary thromboembolic disease. Positron emission tomography and nuclear magnetic resonance imaging were performed to exclude angiosarcoma. At this writing, the patient is under evaluation for thromboendarterectomy.

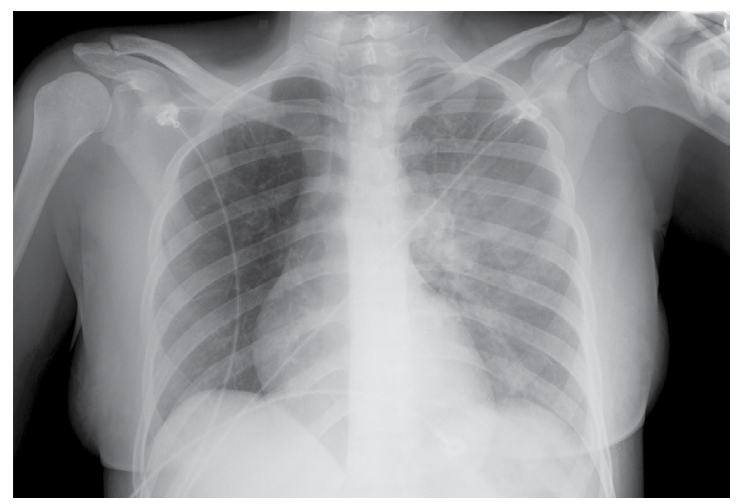

Figure 1. Thoracic $\mathrm{X}$-ray revealing oligemia in the right hemithorax and engorgement of the left pulmonary artery.

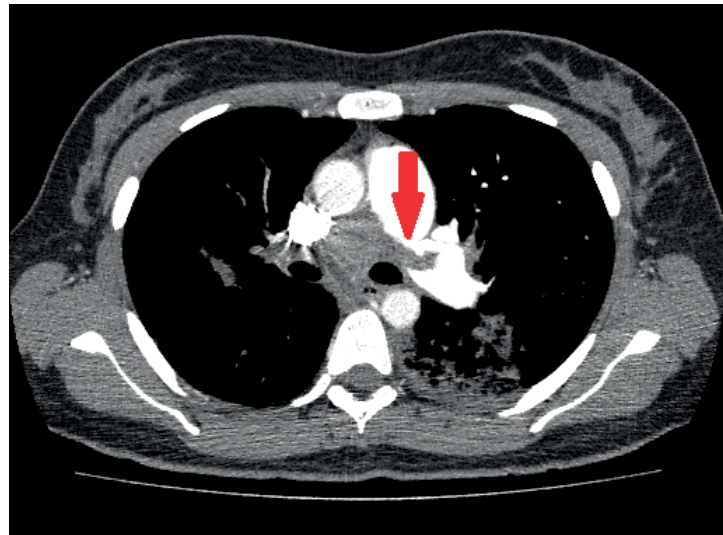

Figure 2. CT scan confirming a thrombus in the pulmonary artery trunk and full occlusion of the right segment (arrow).

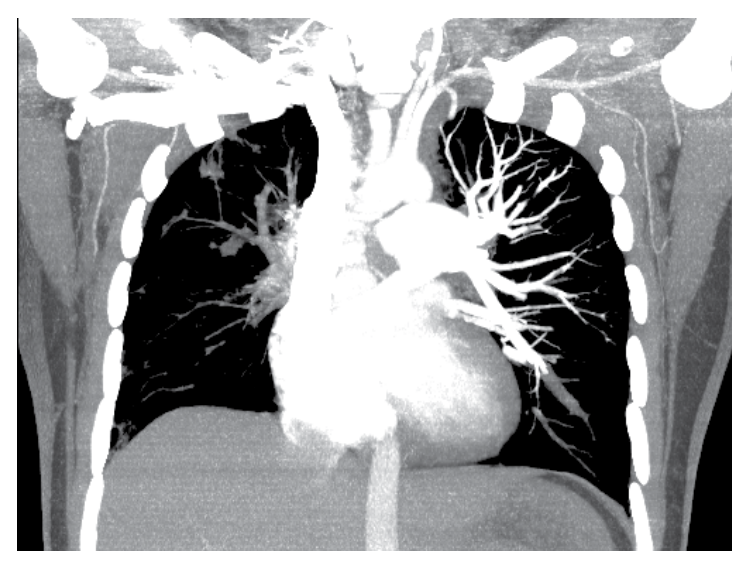

Figure 3. Coronal reconstruction demonstrating the complete lack of pulmonary perfusion in the right lung, together with ipsilateral oligemia. 